\title{
A Comparative X-ray Diffraction Study and $A b$ Initio Calculation on RU60358, a New Pyrethroid
}

\author{
Fodil Hamzaoui ${ }^{1}$, Abdelkader Chouaih ${ }^{1}$, Philippe Lagant ${ }^{2}$, Ouassila Belarbi ${ }^{1}$ and Gérard \\ Vergoten $^{2, *}$
}

1 Laboratoire SEA2M, Département de Chimie, Université de Mostaganem, 27000 Mostaganem, Algeria; E-mail: aek_chouaih@yahoo.fr for Abdelkader Chouaih

2 UMR CNRS 8576 Glycobiologie Structurale et Fonctionnelle, Université des Sciences et Technologies, 59655 Villeneuve d'Ascq, France

* Author to whom correspondence should be addressed; E-mail: Gerard.Vergoten@ univ-lille1.fr Received: 28 February 2006, in Revised Form: 12 April 2006 / Accepted: 30 July 2006 / Published: 9 August 2006

\begin{abstract}
The crystal structure of RU60358, $\mathrm{C}_{20} \mathrm{H}_{21} \mathrm{NO}_{3}$, has been determined using X-ray diffraction to establish the configuration and stereochemistry of the molecule around the C15-C16 triple bond. The compound crystallises in the orthorhombic space group P $2{ }_{1} 2_{1} 2_{1}$, a $=7.7575, \mathrm{~b}=11.3182, \mathrm{c}=21.3529 \AA, \mathrm{V}=1874.80 \AA 3$ and $\mathrm{Z}=4$. The structure has been refined to a final $\mathrm{R}=0.068$ for the observed structure factors with $I \geq 3 \sigma(I)$. The refined structure was found to be significantly non planar. A comparative study, using the $a b$ initio calculations of the structure at B3LYP/6-31G** levels of theory, shows good geometrical agreement with the X-ray diffraction data. Standard deviations between the calculated and experimental bond values have been shown to be $0.01 \AA$ and $0.5^{\circ}$ for bond angles. Vibrational wavenumbers were obtained from a normal mode analysis using the $a b$ initio calculations.
\end{abstract}

Keywords: X-ray diffraction, Ab initio calculation, organic compounds, pyrethroid.

\section{Introduction}

The structures of pyrethroids compounds obtained from experimental X-ray diffraction data has been investigated by several authors [1-4]. Considerable progress has been made in relating the structure of pyrethroids with their biological activity, but improvement of such concepts requires reliable information on molecular shape (configuration, bond lengths and angles and conformation). 
Biological activity in pyrethroids is related to molecular structure and depends strongly on the stereochemistry adopted by the asymmetric centers [5-7]. In this context the compound RU60358 appears as an useful intermediate in the synthesis of some pyrethroid insecticides [8]. The study of the conformation of this molecule may yield information about the mechanism of its biological activity $[9,10]$. In this paper, we propose a comparative study between the experimental X-ray diffraction data and the optimized geometry predicted from ab initio molecular orbital calculations performed on the compound RU60358. Vibrational wavenumbers were then consequently predicted and correct assignments obtained.

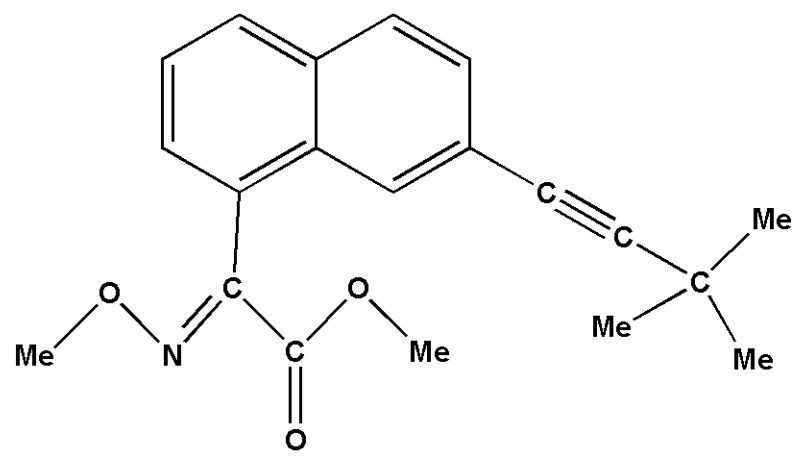

RU60358

\section{Results and discussion}

\subsection{Description of the crystal structure}

The displacement ellipsoid plot with the numbering scheme for the title compound is shown in Figure 1. Figure 2 shows a perspective view of the crystal packing in the unit cell. Selected X-ray diffraction data summarize bond lengths in Table 1 and bond angles in Table 2.

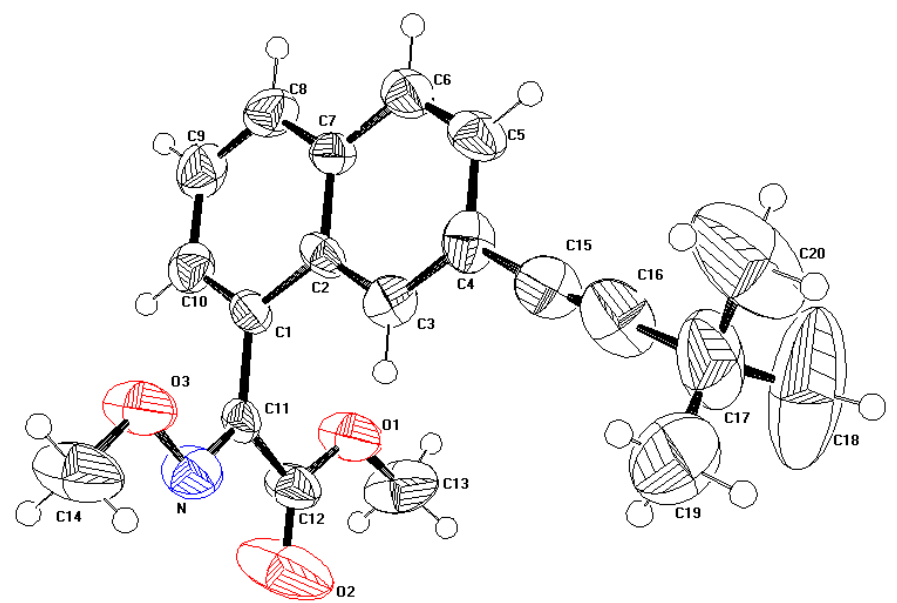

Figure 1. Perspective view of the molecule showing the atom-labelling scheme. Displacement ellipsoids are drawn at the $50 \%$ probability level and $\mathrm{H}$ atoms are shown as small spheres of arbitrary radii. 


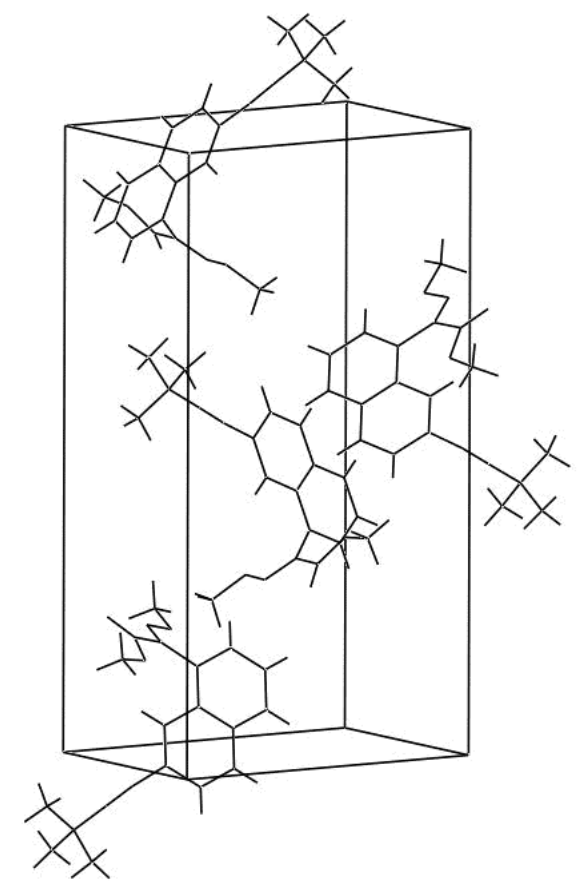

Figure 2. A perspective view of the crystal packing in the unit cell.

Table 1. Selected bond distances $(\AA)$ by X-ray and theoretical calculations

\begin{tabular}{cccc}
\hline \multirow{2}{*}{ Atom 1} & \multirow{2}{*}{ Atom 2} & Distance $(\AA)$ & \\
\cline { 3 - 4 } C1 & C2 & $1.395(10)$ & B3LYP/6-31G** \\
C1 & C10 & $1.395(11)$ & 1.433 \\
C1 & C11 & $1.474(12)$ & 1.381 \\
C2 & C3 & $1.410(09)$ & 1.416 \\
C2 & C7 & $1.395(11)$ & 1.433 \\
C3 & C4 & $1.395(13)$ & 1.388 \\
C4 & C5 & $1.395(10)$ & 1.428 \\
C4 & C15 & $1.429(10)$ & 1.428 \\
C5 & C6 & $1.395(10)$ & 1.371 \\
C6 & C7 & $1.395(09)$ & 1.422 \\
C7 & C8 & $1.395(11)$ & 1.418 \\
C8 & C9 & $1.395(11)$ & 1.375 \\
C9 & C10 & $1.395(09)$ & 1.412 \\
C11 & N & $1.278(13)$ & 1.290 \\
C11 & C12 & $1.514(15)$ & 1.497 \\
C12 & O1 & $1.292(10)$ & 1.351 \\
C12 & O2 & $1.152(10)$ & 1.210 \\
C13 & O1 & $1.439(12)$ & 1.436 \\
N & O3 & $1.376(09)$ & 1.374 \\
C14 & O3 & $1.427(11)$ & 1.467 \\
C15 & C16 & $1.163(13)$ & 1.213 \\
C16 & C17 & $1.467(12)$ & 1.459 \\
C17 & C18 & $1.439(12)$ & 1.548 \\
C17 & C19 & $1.469(11)$ & 1.541 \\
C17 & C20 & $1.531(13)$ & 1.549 \\
\hline
\end{tabular}


Table 2. Selected bond angles $\left(^{\circ}\right)$ by X-ray and theoretical calculations

\begin{tabular}{|c|c|c|c|c|}
\hline \multirow{2}{*}{ Atom 1} & \multirow{2}{*}{ Atom 2} & \multirow{2}{*}{ Atom 3} & \multicolumn{2}{|l|}{ Angle $\left(^{\circ}\right)$} \\
\hline & & & X-ray & B3LYP/6-31G** \\
\hline $\mathrm{C} 2$ & $\mathrm{C} 1$ & C10 & $120.0(8)$ & 119.8 \\
\hline $\mathrm{C} 2$ & $\mathrm{C} 1$ & $\mathrm{C} 11$ & $123.5(5)$ & 121.1 \\
\hline $\mathrm{C} 10$ & $\mathrm{C} 1$ & $\mathrm{C} 11$ & $116.2(8)$ & 119.1 \\
\hline $\mathrm{C} 1$ & $\mathrm{C} 2$ & C3 & $120.2(6)$ & 122.6 \\
\hline $\mathrm{C} 1$ & $\mathrm{C} 2$ & $\mathrm{C} 7$ & $120.0(7)$ & 118.5 \\
\hline $\mathrm{C} 3$ & $\mathrm{C} 2$ & $\mathrm{C} 7$ & $119.8(7)$ & 118.7 \\
\hline $\mathrm{C} 2$ & $\mathrm{C} 3$ & $\mathrm{C} 4$ & $120.0(5)$ & 121.5 \\
\hline $\mathrm{C} 3$ & $\mathrm{C} 4$ & $\mathrm{C} 5$ & $120.0(5)$ & 119.2 \\
\hline $\mathrm{C} 3$ & $\mathrm{C} 4$ & $\mathrm{C} 15$ & $122.1(6)$ & 120.9 \\
\hline C5 & $\mathrm{C} 4$ & $\mathrm{C} 15$ & $117.9(8)$ & 119.9 \\
\hline $\mathrm{C} 4$ & $\mathrm{C} 5$ & C6 & $120.0(8)$ & 120.4 \\
\hline C5 & C6 & $\mathrm{C} 7$ & $120.4(7)$ & 121.2 \\
\hline $\mathrm{C} 2$ & $\mathrm{C} 7$ & C6 & $119.8(9)$ & 118.8 \\
\hline $\mathrm{C} 2$ & $\mathrm{C} 7$ & $\mathrm{C} 8$ & $120.0(5)$ & 119.6 \\
\hline C6 & $\mathrm{C} 7$ & C8 & $120.2(6)$ & 121.6 \\
\hline $\mathrm{C} 7$ & $\mathrm{C} 8$ & $\mathrm{C} 9$ & $120.0(5)$ & 120.6 \\
\hline $\mathrm{C} 8$ & C9 & $\mathrm{C} 10$ & $120.0(7)$ & 120.1 \\
\hline $\mathrm{C} 16$ & $\mathrm{C} 17$ & C19 & $111.5(9)$ & 109.4 \\
\hline $\mathrm{C} 16$ & $\mathrm{C} 17$ & $\mathrm{C} 20$ & $107.7(9)$ & 109.3 \\
\hline C18 & C17 & C19 & $110.5(8)$ & 109.7 \\
\hline $\mathrm{C} 18$ & $\mathrm{C} 17$ & $\mathrm{C} 20$ & $107.3(5)$ & 109.6 \\
\hline C19 & $\mathrm{C} 17$ & $\mathrm{C} 20$ & $107.2(4)$ & 109.5 \\
\hline $\mathrm{C} 1$ & $\mathrm{C} 10$ & C9 & $120.0(6)$ & 121.2 \\
\hline $\mathrm{C} 1$ & $\mathrm{C} 11$ & $\mathrm{~N}$ & $126.9(8)$ & 125.7 \\
\hline $\mathrm{C} 1$ & $\mathrm{C} 11$ & $\mathrm{C} 12$ & $121.6(5)$ & 121.4 \\
\hline $\mathrm{N}$ & $\mathrm{C} 11$ & $\mathrm{C} 12$ & $111.5(4)$ & 112.9 \\
\hline $\mathrm{C} 12$ & $\mathrm{O} 1$ & $\mathrm{C} 13$ & $117.0(9)$ & 115.0 \\
\hline $\mathrm{N}$ & $\mathrm{O} 3$ & $\mathrm{C} 14$ & $108.5(5)$ & 109.2 \\
\hline C11 & $\mathrm{N}$ & $\mathrm{O} 3$ & $110.8(7)$ & 112.6 \\
\hline $\mathrm{C} 11$ & $\mathrm{C} 12$ & O1 & $110.6(7)$ & 110.5 \\
\hline $\mathrm{C} 11$ & $\mathrm{C} 12$ & $\mathrm{O} 2$ & $126.0(9)$ & 125.7 \\
\hline $\mathrm{O} 1$ & $\mathrm{C} 12$ & $\mathrm{O} 2$ & $123.4(5)$ & 123.8 \\
\hline $\mathrm{C} 4$ & C15 & C16 & $174.8(9)$ & 180.5 \\
\hline $\mathrm{C} 15$ & C16 & C17 & $179.3(6)$ & 180.1 \\
\hline $\mathrm{C} 16$ & $\mathrm{C} 17$ & C18 & $112.6(7)$ & 109.6 \\
\hline
\end{tabular}




\subsection{Optimized geometry}

Calculated geometric parameters at B3LYP/6-311G** are equally displayed into Tables 1 and 2. From the theoretical values, it is noteworthy that most of the optimized bond lengths have slightly larger values than the corresponding experimental ones, due to the fact that theoretical calculations imply isolated molecules in gaseous phase state while experimental results refer to molecules in the solid state. Comparing theoretical bond angles with those given in experimental data, the B3LYP calculated values correlate in a good agreement. In spite of the differences, calculated geometric parameters represent a good approximation and can provide a starting point to calculate other parameters, such as vibrational wavenumbers, as will be described below.

\subsection{Vibrational wavenumbers}

The theoretically derived DFT vibrational wavenumbers corresponding to the optimized geometry can be expressed in the more convenient internal coordinate space for a description of the potential energy distribution (PED) via the Redong Program [11]. This algorithm uses least square methods to fit the theoretical vibrational wavenumbers to the experimental ones by introducing scaling factors associated to the various types of force constants.

Table 3 displays the potential energy distribution among internal coordinates obtained for the scaled DFT normal modes analysis (scaling factor $=0.962$ ). This last value is currently recommended to obtain correct vibrational wavenumbers and adequate assignments of the PED [12].

The $v C=\mathrm{N}$ stretching mode of the imine part is generally observed in the $1665-1675 \mathrm{~cm}-1$ range by Raman spectroscopy. The presence of neigbouring aryl groups lowers this value by $10-20 \mathrm{~cm}-1$. Using a general scaling factor of 0.962 leads to the $1594 \mathrm{~cm}^{-1}$ DFT theoretical wavenumber $\left(1657 \mathrm{~cm}^{-1}\right.$ without scaling). This assignment is in accordance with the Raman wavenumbers as reported by Dollish et al. [13]. The imine moiety participates also to Raman bands around $1274 \mathrm{~cm}^{-1}$, presently calculated at $1266 \mathrm{~cm}^{-1}$. Other vibrational modes of interest implying the imine group are predicted to occur at $314 \mathrm{~cm}^{-1}\left(\delta\left(\mathrm{N}-\mathrm{O}-\mathrm{CH}_{3}\right)\right.$, at $971.6 \mathrm{~cm}^{-1}(\mathrm{vN}-\mathrm{O})$ and at $997 \mathrm{~cm}^{-1}$ for the $\left(\mathrm{VO}-\mathrm{CH}_{3}\right)$ stretching mode.

The alkyne $v C \equiv C$ stretching mode displays intense Raman bands in the $2220-2240 \mathrm{~cm}^{-1}$ range. The DFT corresponding wavenumber is obtained presently at $2243 \mathrm{~cm}^{-1}$. Keeping the same 0.962 scaling factor, the $\delta \mathrm{C}-\mathrm{C} \equiv \mathrm{C}$ in plane bending mode is predicted to occur at $474.6 \mathrm{~cm}^{-1}$, this frequency being quite comparable to the experimental range centered at $484 \mathrm{~cm}^{-1}$ [13].

The ester group gives a predicted DFT $v \mathrm{C}=\mathrm{O}$ stretching mode located at $1749 \mathrm{~cm}^{-1}$ corresponding to current experimental bands obtained in the $1730-1750 \mathrm{~cm}^{-1}$ range. The associated $\mathrm{vC}-\mathrm{O}$ stretching mode is predicted at $1215 \mathrm{~cm}^{-1}$, this value appearing quite consistent with experimental data (1200$\left.1220 \mathrm{~cm}^{-1}\right)$. The in plane $\delta(\mathrm{O}-\mathrm{C}=\mathrm{O})$ bending mode is observed in the $750-775 \mathrm{~cm}^{-1}$ range using Raman spectroscopy and the DFT derived corresponding wavenumber is obtained here at $752 \mathrm{~cm}^{-1}$. The $\delta(\mathrm{C}-$ $\mathrm{C}=\mathrm{O})$ and $\delta\left(\mathrm{C}-\mathrm{O}-\mathrm{CH}_{3}\right)$ in plane bending motions are predicted to stand at 374 and $298 \mathrm{~cm}^{-1}$ respectively, these two wavenumbers being very close to the experimental data $\left(300-340 \mathrm{~cm}^{-1}\right)$.

For the aromatic part of the molecule, we can observe a rough accordance between the DFT predicted wavenumbers (and corresponding vibrational assignments) and the experimental data obtained from vibrational analyses on benzene derivatives [14]. The pyrethoid molecule displays numerous and complex mixings of vibrational modes between the two rings and their substituents.

The v8 degenerate (8a, 8b) mode (in Wilson's notation) [14] implying vCA-CA ring stretching 
motions is generally observed around $1596 \mathrm{~cm}^{-1}$. DFT calculations predict contributions from these two modes at 1558 and in the $1582-1594 \mathrm{~cm}^{-1}$ range.

$v \mathrm{CA}-\mathrm{CA}$ and $\delta \mathrm{CH}$ modes related to the $\delta_{19}$ degenerate mode [14] give rise to experimental vibrational bands around $1485 \mathrm{~cm}^{-1}$. The DFT derived $1484.6 \mathrm{~cm}^{-1}$ wavenumber corresponds correctly to such an assignment.

The mode 4 of benzene generally gives rise to weak Raman and strong IR bands. This out of plane mode $(\gamma \mathrm{CH})$ is generally observed to occur around $693 \mathrm{~cm}^{-1}$. The DFT wavenumber obtained presently at $738 \mathrm{~cm}^{-1}$ could correspond to this mode.

The intense vCA-CA ( $v_{1}$ in Wilson's notation) ring stretching mode appears in Raman spectra at $992 \mathrm{~cm}^{-1}$. This mode has been identified as being slightly dependent of $\delta \mathrm{CH}$ bending motions. Due to large mixings of vibrational motions taking place between the two rings, an attempt in the assignment of this mode from DFT calculation is given here at $971.6 \mathrm{~cm}^{-1}$.

From DFT vibrational analyses, out of plane $\gamma \mathrm{CH}$ motions display in and out of phase motions depending on the ring. These modes are predicted at 819 and $825 \mathrm{~cm}^{-1}$. Other contributions to the out of plane motions have been also obtained theoretically at 887 and $933 \mathrm{~cm}^{-1}$. An attempt to assign these wavenumbers to the $v 5,10 \mathrm{a}$ and $10 \mathrm{~b}$ normal modes as observed for benzene derivatives [13-15] remains however difficult.

Table 3. Normal modes analysis of RU60358 from DFT (RB3LYP/6-31G (d, p).

\begin{tabular}{|c|c|c|c|}
\hline$v\left(\mathrm{~cm}^{-1}\right)^{*}$ & Assignments & $v\left(\mathrm{~cm}^{-1}\right)^{*}$ & Assignments \\
\hline 10.6 & $\tau_{\mathrm{C} 17 \mathrm{CT},}, \tau_{\mathrm{C} 1 \mathrm{C} 11}, \delta_{\mathrm{C} 4, \mathrm{C} 15, \mathrm{C} 16}$ & 702.1 & $v_{\mathrm{CACA}}, v_{\mathrm{ClC} 11}, \delta_{\mathrm{CACACA}}, \delta_{\mathrm{CT}, \mathrm{O}, \mathrm{N}}$ \\
\hline 13.8 & $\tau_{\mathrm{C} 1 \mathrm{C} 11}$ & 723.4 & $v \mathrm{~s}_{\mathrm{CT}(\mathrm{CH} 3) 3(\mathrm{tBu})}$ \\
\hline 24.7 & $\tau_{\mathrm{C} 11 \mathrm{C} 12}$ & 738.1 & $\gamma_{\mathrm{CAH}}, \gamma_{\mathrm{CACA}}, \tau_{\mathrm{CACA}}$ \\
\hline 30.9 & $\delta_{\mathrm{CA}, \mathrm{C} 4, \mathrm{C} 15}$ & 749.6 & $\gamma_{\mathrm{C} 12=\mathrm{O} 2}, \gamma_{\mathrm{C} 11 \mathrm{~N}}, \delta_{\mathrm{C} 12=\mathrm{O} 2}$ \\
\hline 35.4 & $\tau_{\mathrm{C} 4 \mathrm{C} 15}, \tau_{\mathrm{C} 1 \mathrm{C} 2}, \delta_{\mathrm{C} 15, \mathrm{C} 16, \mathrm{C} 17}$ & 752.2 & $\gamma_{\mathrm{C} 12=\mathrm{O} 2}, \delta_{\mathrm{C} 12=\mathrm{O} 2}, \delta_{\mathrm{C} 12 \mathrm{O} 1 \mathrm{CT}}, v_{\mathrm{C} 12 \mathrm{O} 1}$ \\
\hline 61.9 & $\tau_{\mathrm{C} 1 \mathrm{C} 11}, \tau_{\mathrm{C} 1 \mathrm{CA}}, \tau_{\mathrm{C} 4 \mathrm{C} 15}$ & 778.7 & $\gamma_{\mathrm{CAH}}, \gamma_{\mathrm{C} 1 \mathrm{C} 11}, \gamma_{\mathrm{CACA}}$ \\
\hline 67.2 & $\tau_{\mathrm{NO}}, \gamma_{\mathrm{C} 11 \mathrm{~N}}$ & 819.0 & $\gamma_{\mathrm{CAH}}, \gamma_{\mathrm{CACA}}$ \\
\hline 98.1 & $\tau_{\mathrm{C} 14 \mathrm{O}}, \tau_{\mathrm{NO}}$ & 825.3 & $\delta_{\mathrm{CACACA}(\text { ring } 1)}, \gamma_{\mathrm{CAH}} \#, v_{\mathrm{CACA}}$ \\
\hline 102.4 & $\delta_{\mathrm{C} 15, \mathrm{C} 16, \mathrm{C} 17}, \tau_{\mathrm{C} 6 \mathrm{C} 7}$ & 832.4 & $\delta_{\mathrm{CACACA}}, v_{\mathrm{C} 16 \mathrm{C} 17}, v_{\mathrm{C} 17 \mathrm{CT}}$ \\
\hline 108.4 & $\delta_{\mathrm{C} 12, \mathrm{C} 11, \mathrm{~N}}, \tau_{\mathrm{O} 1 \mathrm{C} 13}, \delta_{\mathrm{Cl} 1 \mathrm{C} 11, \mathrm{C} 12}$ & 873.0 & $\gamma_{\mathrm{C} 3 \mathrm{H}}, \tau_{\mathrm{CACA}}$ \\
\hline 112.8 & $\tau_{\mathrm{O} 1 \mathrm{C} 13}$ & 887.1 & $\gamma_{\mathrm{CAH}(\text { ring 1) }}$ \\
\hline 129.4 & $\delta_{\mathrm{C} 4, \mathrm{C} 15, \mathrm{C} 16}, \tau_{\mathrm{O} 1 \mathrm{C} 12}$ & 897.4 & $v_{\mathrm{O} 3 \mathrm{~N}}, v_{\mathrm{O} 3 \mathrm{CT}}, v_{\mathrm{O} 1 \mathrm{C} 12}, v_{\mathrm{O} 1 \mathrm{CT}}, v_{\mathrm{C} 17 \mathrm{CT}}$ \\
\hline 158.9 & $\tau_{\mathrm{C} 2 \mathrm{C} 7}, \tau_{\mathrm{C} 2 \mathrm{C} 3}, \tau_{\mathrm{C} 7 \mathrm{C} 8}$ & 897.8 & $v_{\mathrm{C} 17 \mathrm{CT}}, \rho_{(\mathrm{BBu})}$ \\
\hline 163.6 & $\tau_{\mathrm{O} 1 \mathrm{C} 12}, \tau_{\mathrm{O} 3 \mathrm{C} 14}, \tau_{\mathrm{ON}}$ & 899.7 & $v_{\mathrm{C} 17 \mathrm{CT}}$ \\
\hline 173.9 & $\tau_{\mathrm{ON},} \delta_{\mathrm{C} 4, \mathrm{C} 15, \mathrm{C} 16}, \tau_{\mathrm{O} 3 \mathrm{C} 14}$ & 926.0 & $v_{\mathrm{C} 17 \mathrm{CT}}, v_{\mathrm{NO} 3}, v_{\mathrm{C} 4 \mathrm{CA}}, \delta_{\mathrm{CACACA}}$ \\
\hline 185.2 & $\tau_{\mathrm{C} 6 \mathrm{C} 7}, \tau_{\mathrm{C} 1 \mathrm{C} 2}, \tau_{\mathrm{C} 4 \mathrm{C} 15}$ & 933.0 & $\gamma_{\mathrm{CAH}(\text { all rings })}$ \\
\hline 218.6 & $v_{\mathrm{C} 4 \mathrm{C} 15}, v_{\mathrm{C} 16 \mathrm{C} 17}, \delta_{\mathrm{C} 3, \mathrm{C} 4, \mathrm{C} 5}$ & 933.8 & $\rho_{(\mathrm{tBu})}$ \\
\hline 221.9 & $\tau_{\mathrm{C} 17 \mathrm{CH} 3(\mathrm{BBu})}$ & 942.9 & $\gamma_{\mathrm{CAH}(\text { all rings) }}$ \\
\hline 246.0 & $\delta_{\mathrm{C} 12, \mathrm{O} 1, \mathrm{C} 13}, \delta_{\mathrm{O} 1, \mathrm{C} 12, \mathrm{C} 11}, \tau_{\mathrm{C} 4 \mathrm{C} 15}$ & 971.6 & $v_{\mathrm{O} 3 \mathrm{~N}}, v_{\mathrm{CACA}}, \delta_{\mathrm{CACACA}}, \delta_{\mathrm{CAH}}, \delta_{\mathrm{C} 11, \mathrm{~N}, \mathrm{O} 3}$ \\
\hline 269.5 & $\tau_{\mathrm{C} 17 \mathrm{CH} 3(\mathrm{BBu})}$ & 996.9 & $v_{\mathrm{CTO} 1}, v_{\text {ОЗСТ }}$ \\
\hline 276.5 & $\tau_{\mathrm{C} 17 \mathrm{CH} 3} \quad$ (tBu) & 1013.9 & $\rho_{(\mathrm{Bu})}$ \\
\hline 279.4 & $\delta_{\mathrm{C} 12, \mathrm{O} 1, \mathrm{C} 13}, v_{\mathrm{C} 1 \mathrm{C} 11}, \delta_{\mathrm{C} 1, \mathrm{C} 11, \mathrm{C} 12}$ & 1014.1 & $\rho_{(\mathrm{tBu})}$ \\
\hline 297.8 & $\tau_{\mathrm{C} 11 \mathrm{~N}}, \delta_{\mathrm{C} 12, \mathrm{O} 1, \mathrm{C} 13}, \tau_{\mathrm{O} 1 \mathrm{C} 12}$ & 1045.1 & $v_{\mathrm{CACA}}, v_{\mathrm{O} 3 \mathrm{CT}}, v_{\mathrm{O} 3 \mathrm{~N}}$ \\
\hline 314.3 & $\delta_{\mathrm{C} 14, \mathrm{O}, \mathrm{N}}, v_{\mathrm{C} 1 \mathrm{C} 11}, \tau_{\mathrm{C} 11 \mathrm{~N}}, \delta_{\mathrm{CACACA}}$ & 1060.2 & $v_{\mathrm{O} 3 \mathrm{CT}}, v_{\mathrm{CACA}}, v_{\mathrm{O} 3 \mathrm{~N}}$ \\
\hline 332.4 & $\delta_{\mathrm{CT}, \mathrm{C} 17, \mathrm{CT}(\mathrm{tBu})}$ & 1095.2 & $v_{\mathrm{C} 12 \mathrm{O} 1}, v_{\mathrm{CTO} 1}, \delta_{\mathrm{C} 11, \mathrm{~N}, \mathrm{O} 3}, v_{\mathrm{O} 3 \mathrm{CT}}$ \\
\hline 336.7 & $\delta_{\mathrm{CT}, \mathrm{C} 17, \mathrm{CT}(\mathrm{BBu})}$ & 1130.9 & $\rho_{\mathrm{C} 14 \mathrm{H} 3}$ \\
\hline 357.3 & $\delta_{\mathrm{CT}, \mathrm{C} 17, \mathrm{CT}(\mathrm{tBu})}, \delta_{\mathrm{C} 1, \mathrm{C} 2, \mathrm{C} 3}, \delta_{\mathrm{C} 6, \mathrm{C} 7, \mathrm{C} 8}$ & 1133.8 & $\rho_{\mathrm{C} 13 \mathrm{H} 3}$ \\
\hline
\end{tabular}


Table 3. (continued)

\begin{tabular}{|c|c|c|c|}
\hline 367.4 & $v_{\mathrm{C} 11 \mathrm{C} 12}, \delta_{\mathrm{C} 11, \mathrm{~N}, \mathrm{O}}, \delta_{\mathrm{C} 12, \mathrm{C} 11, \mathrm{~N}}$ & 1134.5 & $\delta_{\mathrm{CAH}}, \boldsymbol{v}_{\mathrm{CACA}(\text { ring } 2)}$ \\
\hline 373.6 & $\delta_{\mathrm{C} 11, \mathrm{C} 12, \mathrm{O} 2}, \delta_{\mathrm{C} 12, \mathrm{O} 1, \mathrm{C} 13}$ & 1148.6 & $\delta_{\mathrm{CAH}}, v_{\mathrm{CACA}(\text { ring } 1)}$ \\
\hline 387.9 & $\tau_{\mathrm{C} 4 \mathrm{C} 15}, \tau_{\mathrm{C} 4 \mathrm{CA}}, \gamma_{\mathrm{C} 6 \mathrm{C} 7}$ & 1163.1 & $\rho_{\mathrm{C} 13 \mathrm{H} 3}$ \\
\hline 424.2 & $\delta_{\mathrm{CT}, \mathrm{C} 17, \mathrm{CT}(\mathrm{tBu})}$ & 1172.1 & $\rho_{\mathrm{C} 14 \mathrm{H} 3}$ \\
\hline 425.2 & $\tau_{\mathrm{CACA}}, \gamma_{\mathrm{CAH}}$ & 1181.6 & $\rho_{(\mathrm{tBu})}$ \\
\hline 474.6 & $\delta_{\mathrm{C} 4, \mathrm{C} 15, \mathrm{C} 16}, \delta_{\mathrm{C} 16, \mathrm{C} 17, \mathrm{CT}}, \gamma_{\mathrm{C}-\mathrm{N}}$ & 1183.4 & $\rho_{(\mathrm{tBu})}$ \\
\hline 501.5 & $\delta_{\mathrm{C} 16, \mathrm{C} 17, \mathrm{CT}}, \delta_{\mathrm{CA}, \mathrm{C} 4, \mathrm{C} 15}, \delta_{\mathrm{CACACA}}$ & 1184.8 & $v_{\mathrm{O} 1 \mathrm{C} 12,} \rho_{\mathrm{C} 14 \mathrm{H} 3}, v_{\mathrm{C} 4 \mathrm{C} 15}, \rho_{\mathrm{C} 13 \mathrm{H} 3}, \rho_{(\mathrm{tBu})}$ \\
\hline 512.7 & $\delta_{\mathrm{C} 16, \mathrm{C} 17, \mathrm{CT}}, \delta_{\mathrm{C} 15, \mathrm{C} 16, \mathrm{C} 17}, \tau_{\mathrm{CACA}}$ & 1196.4 & $v_{\mathrm{CACA}}, \delta_{\mathrm{CAH}}$ \\
\hline 528.1 & $\gamma_{\mathrm{CACA}}, \tau_{\mathrm{CACA}}, \gamma_{\mathrm{C} 1 \mathrm{C} 11}, \delta_{\mathrm{C} 14, \mathrm{O}, \mathrm{N}}$ & 1215.3 & $\delta_{\mathrm{CAH}}, v_{\mathrm{O} 1 \mathrm{C} 12}$ \\
\hline 545.3 & $\tau_{\mathrm{C} 4 \mathrm{C} 15}, \delta_{\mathrm{C} 15, \mathrm{C} 16, \mathrm{C} 17}, \delta_{\mathrm{C} 16, \mathrm{C} 17, \mathrm{CT}}, \tau_{\mathrm{CACA}}, \gamma_{\mathrm{C} 4 \mathrm{C} 15}$ & 1241.2 & $\delta_{\mathrm{CAH}(\text { ring 2) }}$ \\
\hline 550.4 & $\delta_{\mathrm{C} 4, \mathrm{C} 15, \mathrm{C} 16}, \delta_{\mathrm{C} 16, \mathrm{C} 17, \mathrm{CT}}$ & 1266.0 & $v_{\mathrm{C} 11 \mathrm{C} 12}, v_{\mathrm{C} 1 \mathrm{C} 11}, v_{\mathrm{O} 1 \mathrm{C} 12}, \delta_{\mathrm{CAH}(\text { ring } 1)}$ \\
\hline 583.3 & $\tau_{\mathrm{C} 11 \mathrm{~N}}, v_{\mathrm{C} 16 \mathrm{C} 17}, \delta_{\mathrm{CA}, \mathrm{C} 1, \mathrm{C} 11}, \gamma_{\mathrm{C} 11 \mathrm{~N}}, \gamma_{\mathrm{C} 12=\mathrm{O} 2}$ & 1270.9 & $v_{\mathrm{C} 16 \mathrm{C} 17}, v_{\mathrm{C} 4 \mathrm{C} 15}, \delta_{\mathrm{CAH}(\text { ring } 1)}$ \\
\hline 590.2 & $\gamma_{\mathrm{C} 11 \mathrm{~N}}, \tau_{\mathrm{C} 11 \mathrm{C} 12}, \gamma_{\mathrm{C} 12=02}, \gamma_{\mathrm{C} 4 \mathrm{C} 15}$ & 1303.8 & $v_{\mathrm{CACA}}, \delta_{\mathrm{CAH}}$ \\
\hline 616.1 & $\delta_{\mathrm{CACACA}}, \delta_{\mathrm{CT}, \mathrm{O}, \mathrm{N}}, v_{\mathrm{C} 17 \mathrm{CT}}$ & 1352.3 & $v_{\mathrm{CACA}}$ \\
\hline 652.7 & $\gamma_{\mathrm{C} 4 \mathrm{C} 15}, \gamma_{\mathrm{C} 1 \mathrm{C} 11}, \tau_{\mathrm{CACA}}, \gamma_{\mathrm{CAH}}$ & 1353.7 & $\delta \mathrm{s}_{\mathrm{CH} 3(\mathrm{tBu})}$ \\
\hline 1354.4 & $\delta \mathrm{s}_{\mathrm{CH} 3(\mathrm{tBu})}$ & 2243.4 & $v_{\mathrm{C} 15 \mathrm{C} 16}$ \\
\hline 1364.7 & $v_{\mathrm{CACA}}, \delta_{\mathrm{CAH}}$ & 2925.2 & $\mathrm{~V}_{\mathrm{sCH} 3(\mathrm{tBu})}$ \\
\hline 1384.7 & $\delta_{\mathrm{sCH} 3(\mathrm{tBu})}$ & 2925.8 & $\mathrm{~V}_{\mathrm{sCH} 3(\mathrm{tBu})}$ \\
\hline 1416.2 & $\delta_{\mathrm{sC} 14 \mathrm{H} 3}$ & 2931.6 & $v_{\mathrm{sC} 14 \mathrm{H} 3}$ \\
\hline 1421.4 & $\delta_{\mathrm{sC} 13 \mathrm{H} 3}$ & 2931.8 & $v_{\mathrm{sC} 14 \mathrm{H} 3}, v_{\mathrm{S}_{\mathrm{CH} 3}(\mathrm{tBu})}$ \\
\hline 1423.3 & $\delta_{\mathrm{sC} 13 \mathrm{H} 3}$ & 2947.1 & $\mathrm{~V}_{\mathrm{sC} 13 \mathrm{H} 3}$ \\
\hline 1433.6 & $\delta_{\mathrm{aCH} 3(\mathrm{tBu})}$ & 2996.8 & $\mathrm{~V}_{\mathrm{aCH} 3(\mathrm{tBu})}$ \\
\hline 1434.7 & $\delta_{\mathrm{aC} 14 \mathrm{H} 3}$ & 2997.4 & $\mathrm{~V}_{\mathrm{aCH} 3(\mathrm{BBu})}$ \\
\hline 1436.2 & $v_{\mathrm{CACA}}, \delta_{\mathrm{CAH}}$ & 3002.5 & $\mathrm{~V}_{\mathrm{aCH} 3(\mathrm{tBu})}$ \\
\hline 1437.8 & $\delta_{\mathrm{aC} 13 \mathrm{H} 3}$ & 3006.2 & $\mathrm{~V}_{\mathrm{aC} 14 \mathrm{H} 3}$ \\
\hline 1443.9 & $\delta_{\mathrm{aCH} 3(\mathrm{tBu})}$ & 3006.8 & $\mathrm{~V}_{\mathrm{aCH} 3(\mathrm{tBu})}$ \\
\hline 1444.4 & $\delta_{\mathrm{aCH} 3(\mathrm{tBu})}$ & 3010.2 & $\mathrm{~V}_{\mathrm{aCH} 3(\mathrm{tBu})}$ \\
\hline 1451.5 & $\delta_{\mathrm{aC} 13 \mathrm{H} 3}$ & 3010.9 & $\mathrm{~V}_{\mathrm{aCH} 3(\mathrm{tBu})}$ \\
\hline 1456.3 & $\delta_{\mathrm{aCH} 3(\mathrm{BBu})}$ & 3020.7 & $\mathrm{~V}_{\mathrm{aC} 13 \mathrm{H} 3}$ \\
\hline 1456.8 & $\delta_{\mathrm{aCH} 3(\mathrm{BBu})}$ & 3041.2 & $\mathrm{~V}_{\mathrm{aC} 14 \mathrm{H} 3}$ \\
\hline 1457.4 & $\delta_{\mathrm{aC} 14 \mathrm{H} 3}$ & 3054.8 & $\mathrm{~V}_{\mathrm{aC} 13 \mathrm{H} 3}$ \\
\hline 1474.6 & $\delta_{\mathrm{aCH} 3(\mathrm{tBu})}$ & 3056.3 & $v_{\mathrm{CAH}(\text { all rings) }}$ \\
\hline 1484.6 & $v_{\mathrm{CACA}}, \delta_{\mathrm{CAH}}$ & 3059.4 & $v_{\mathrm{CAH}(\text { all rings) }}$ \\
\hline 1557.7 & $v_{\mathrm{CACA}}, \delta_{\mathrm{CAH}}$ & 3068.8 & $\mathrm{~V}_{\mathrm{CAH}(\text { ring } 1)}$ \\
\hline 1582.1 & $v_{\mathrm{C} 11 \mathrm{~N}}, v_{\mathrm{CACA}}$ & 3083.6 & $\mathrm{~V}_{\mathrm{CAH}(\text { ring } 1)}$ \\
\hline 1593.9 & $v_{\mathrm{C} 11 \mathrm{~N},} v_{\mathrm{CACA}}$ & 3087.9 & $\mathrm{v}_{\mathrm{CAH}(\text { ring 2) }}$ \\
\hline 1609.1 & $v_{\mathrm{CACA}}$ & 3096.9 & $\mathrm{v}_{\mathrm{CAH}(\text { ring 2) }}$ \\
\hline 1749.0 & $v_{\mathrm{C} 12=\mathrm{O} 2}$ & & \\
\hline
\end{tabular}

$(\mathrm{tBu})$ : Tertiobutyl group. ring 1 is defined by atoms $\mathrm{C}_{1}, \mathrm{C}_{2}, \mathrm{C}_{7}, \mathrm{C}_{8}, \mathrm{C}_{9}$ and $\mathrm{C}_{10}$. ring 2 is defined by atoms $\mathrm{C}_{2}, \mathrm{C}_{3}, \mathrm{C}_{4}, \mathrm{C}_{5}, \mathrm{C}_{6}$ and $\mathrm{C}_{7}$

\# $\mathrm{CA}-\mathrm{H}$ out of plane bending (in phase in each ring but out of phase between rings)

\# CA-H out of plane bending in phase for both rings. $v$ : stretching modes (vs : symmetric, va : antisymmetric)

$\delta$ : in plane valence angle bending, ( $\delta$ s : symmetric, $\delta$ a : antisymmetric). $\tau, \gamma, \rho:$ torsion, out of plane wagging and rocking deformations respectively

(*scaled vibrational frequencies originate from application of a general scaling factor (0.962) applied to all types of internal force constants). 


\section{Experimental Section}

\section{$3.1 X$-ray structure determination}

A needle-shaped crystal of dimensions $0.32 \times 0.27 \times 0.10 \mathrm{~mm}$ was mounted on a Philips Enraf Nonius four-circle diffractometer. Data were collected with MoK $\alpha$ radiation. Intensities for 3082 reflections were measured by the $\omega / 2 \theta$ scan method, scan width $=1.4^{\circ}$, scan speed $0.02^{\circ} \mathrm{s}-1$ and $0<\mathrm{h}$ $<10,0<1<15,0<\mathrm{k}<30$. Lattice parameters from 25 reflections $(2<\theta<30)$. Three standard reflections monitored every two hours, no significant variation was observed. The data were corrected for Lorentz and Polarization effects, but not for absorption or extinction. The structure was determined by considering 1583 reflections with $I \geq 3 \sigma(I)$.

The structure was solved by direct methods implemented in Shelxs [16]. A Fourier synthesis revealed the complete structure, which was refined by full-matrix least squares. All non-H atoms refined anisotropically. The $\mathrm{H}$ atoms were located from a difference Fourier map and included in the refinement with the isotropic temperature factor of the carrier atom. Fig. 1 shows the atomic numbering of the molecule.

The final least-squares cycle using Shelxl [17] gave $\mathrm{R}=0.068$ for all reflections with $I \geq 3 \sigma(I)$, $\mathrm{wR}=6.8 \%, \mathrm{~S}=1.25, \mathrm{w}=1 .(\Delta \rho)_{\min }=-0.271 \mathrm{e}^{-3},(\Delta \rho)_{\max }=0.380 \mathrm{e}^{-3}$.

Atomic scattering factors for heavy atoms were taken from International Tables for X-ray Crystallography [18] while the factors for H were those of Stewart, Davidson \& Simpson [19]. A summary of the key crystallographic information is given in Table 4.

Crystallographic data has been deposited with the Cambridge Crystallographic Data Centre with the deposition number CCDC 272 478. These data can be obtained free of charge from The Cambridge Crystallographic Data Centre via www.ccdc.cam.ac.uk/data_request/cif.

\subsection{Computational methods}

Molecular modeling, geometry optimizations, harmonic wavenumbers for the normal modes of vibration and force field in cartesian coordinates were calculated on RU60358 using the Density Functional Theory and the B3LYP [20,21] (Becke's three parameter Hybrid Functional using the LYP correlation Functional) functional together with the 6-31G(d,p) basis set. Such combination is being used with good results for organic molecules [22] and hydrogen-bonded systems [23-25], and represents a good compromise between economy of computational resources, accuracy and applicability to many-atoms molecules. All the calculations were made with the Gaussian 03 set of programs [26]. Ab initio geometry optimization on RU60358 was performed starting from the geometry found in the X-ray refinement. 
Table 4. Crystallographic data and experimental details for $\mathrm{C}_{20} \mathrm{H}_{21} \mathrm{NO}_{3}$

\begin{tabular}{|c|c|}
\hline \multicolumn{2}{|l|}{ Crystal data } \\
\hline Formula & $\mathrm{C}_{20} \mathrm{H}_{21} \mathrm{NO}_{3}$ \\
\hline Molecular weight & 323.34 \\
\hline Crystal system & Orthorhombic \\
\hline Space group & $\mathrm{P} 2{ }_{1} 2_{1} 2_{1}$ \\
\hline Unit cell determination & Least-squares fit from 25 reflections $\left(2^{\circ}<\theta<30^{\circ}\right)$ \\
\hline$a(\AA)$ & 7.7575 \\
\hline$b(\AA)$ & 11.3182 \\
\hline$c(\AA)$ & 21.3529 \\
\hline$V\left(\AA^{3}\right)$ & 1874.80 \\
\hline $\mathrm{Z}$ & 4 \\
\hline$d_{\text {calc }}\left(\mathrm{g} \cdot \mathrm{cm}^{-3}\right)$ & 1.16 \\
\hline$m u\left(\mathrm{~mm}^{-1}\right)$ & 0.077 \\
\hline Crystal colour & Colourless \\
\hline Crystal size & $0.32 \times 0.27 \times 0.10(\mathrm{~mm})$ \\
\hline \multicolumn{2}{|l|}{ Experimental data } \\
\hline \multirow[t]{3}{*}{ Technique } & $\begin{array}{l}\text { Four circles diffractometer, CAD4 Enraf Nonius } \\
\text { kappa geometry }\end{array}$ \\
\hline & Graphite oriented monochromator : Mok $\alpha$ \\
\hline & $\lambda=0.71070 \AA, \omega / 2 \theta$ scan \\
\hline Scanning range for $\theta$ & $2.04-29.96$ \\
\hline Number of reflections measured & 3082 \\
\hline Number of reflections observed & $1583(\mathrm{I} \geq 3 \sigma(\mathrm{I})$ criterion $)$ \\
\hline \multirow[t]{3}{*}{ Limiting indices } & $h \quad 0 \rightarrow 10$ \\
\hline & $k \quad 0 \rightarrow 15$ \\
\hline & $l \quad 0 \rightarrow 30$ \\
\hline$T(\mathrm{~K})$ & 293 \\
\hline \multicolumn{2}{|l|}{ Refinement data } \\
\hline Refinement method & Full-matrix least-squares on $\mathrm{F}$ \\
\hline Final $R$ indices & $\mathrm{R}=0.068, \mathrm{wR}=0.068$ \\
\hline S & 1.249 \\
\hline $\mathrm{H}$ atoms & constrained refinement \\
\hline Parameters & 224 \\
\hline$(\Delta / \sigma)_{\max }$ & 0.380 \\
\hline$(\Delta \rho)_{\min }$ & $-0.271 \mathrm{e}^{-3}$ \\
\hline$(\Delta \rho)_{\max }$ & $0.380 \mathrm{e}^{-3}$ \\
\hline Extinction & No extinction correction applied \\
\hline
\end{tabular}

\section{References}

1. Baert, F.; Guelzim, A. X-ray Structure of the Pyrethroid Insecticide $\left\{1 \mathrm{R}-\left[1 \alpha\left(\mathrm{S}^{*}\right), 2 \alpha\right]\right\}-2-(2,2-$ Dichlorovinyl)-3,3-dimethylcyclopropanecarboxylic Acid Cyano(3-phenoxyphenyl)methyl Ester (RU 24501). Acta Cryst. C 1991, 47, 606-608.

2. Baert, F.; Guelzim, A.; Germain, G. Structure of Two Pyrethroid Insecticides: Acrynathryn (RU 38702) and a Derivative (RU 38181). Acta Cryst. C 1991, 47, 768-771. 
3. Hamzaoui, F.; Lamiot, J.; Baert, F. X-ray Structure of a New Pyrethroid RU 52259. Acta Cryst. C 1993, 49, 818-820.

4. Hamzaoui, F.; Baert, F. A New Pyrethroid Insecticide RU41414. Acta Cryst. C, 1996, 52, 689-690.

5. Tessier, J. Recent Advances in the Chemistry of Insect Control; Janes, N. F., Ed.; The Royal Society of Chemistry, London, 1985, pp. 26-52.

6. Tessier, J.; Teche, A.; Demoute, J. P. Pesticide Chemistry: Human Welfare and the Environment; Miyamoto, J.; Kearney, P. C., Ed.; Pergamon Press: Oxford, New York, 1983; Vol. 1, pp 95-100.

7. Tessier, J.; Teche, A.; Demoute, J. P. Proceedings of the $5^{\text {th }}$ IUPAC International Congress of Pesticide Chemistry; Miyamoto, J.; Kearney, P. C., Ed.; Pergamon Press: Oxford, New York, 1983; pp 197-202.

8. Babin, D.; Demassey, J.; Demoute, J. P.; Dutheil, P.; Terrie I.; Tessier, J. A New Way toward Z $\alpha, \beta$ Unsaturated Esters: A Pyrethroid Application. J. Org. Chem. 1992, 57, 584-589.

9. Elliott, M. The Relationship between the Structure and the Activity of Pyrethroids. Bull. Wld Hlth Org. 1970, 44, 315-324.

10. Elliott, M.; Farnham, A. W.; Janes, N. F.; Needham, P. H.; Pulman, D. A. Insecticidal Activity of the Pyrethrins and Related Compounds. Pestic. Sci. 1975, 6, 537-542.

11. Allouche, A.; Pourcin, J. Ab initio calculation of vibrational force fields: Determination of nonredundant symmetry coordinates by least-square component analysis. Spectrochim. Acta, Part A 1993, 49, 571-580.

12. Pulay, P. Possibilities and limitations of ab initio calculation of vibrational spectra. J. Mol. Struct. 1995, 347, 293-308.

13. Dollish, F. R.; Fateley, W. G.; Bentley, F. F. Characteristic Raman Frequencies of Organic Compounds; John Wiley\& Sons: New York, 1974.

14. Varsányi, G.; Szöke, S. Vibrational Spectra of Benzene Derivatives; Academic Press: New York, 1969.

15. Wilson, E. B., Jr. The Normal Modes and Frequencies of Vibration of the Regular Plane Hexagon Model of the Benzene Molecule. Phys. Rev. 1934, 45, 706-714.

16. Sheldrick, G. M. SHELXS97: Program for crystal structure determination; University of Göttingen, Germany, 1997.

17. Sheldrick, G. M. SHELXL97: Program for crystal structure determination; University of Göttingen, Germany, 1997.

18. Brown, P. J.; Fox, A. G.; Maslen, E. N.; O'Keefe, M. A.; Willis, B. T. M. International Tables for X-ray Crystallography; Wilson, A. J. C.; Prince, E., Ed.; Kluwer Academic Publishers: Dordrecht, 1999; Vol. C, pp 548-589.

19. Stewart, R. F.; Davidson, E. R.; Simpson, W. T. Coherent X-Ray Scattering for the Hydrogen Atom in the Hydrogen Molecule. J. Chem. Phys. 1965, 42, 3175-3187.

20. Becke, A. D. Density-functional thermochemistry. III. The role of exact exchange. J. Chem. Phys. 1993, 98, 5648-5652.

21. Lee, C.; Yang, W.; Parr, R. G. Development of the Colle-Salvetti correlation-energy formula into a functional of the electron density. Phys. Rev. B 1988, 37, 785-789.

22. Rauhut, G.; Pulay, P. Transferable scaling factors for density functional derived vibrational force fields. J. Phys. Chem. 1995, 99, 3093-3100. 
23. Gómez Marigliano, A. C.; Varetti, E. L. Self-Association of Formamide in Carbon Tetrachloride Solutions: An Experimental and Quantum Chemistry Vibrational and Thermodynamic Study. $J$. Phys. Chem. A, 2002, 106, 1100-1106.

24. Gómez Marigliano, A. C.; Varetti, E. L. Unpublished results.

25. Sim, F.; St.-Amant, A.; Papai, I.; Salahub, D. R. Gaussian density functional calculations on hydrogen-bonded systems. J. Am. Chem. Soc. 1992, 114, 4391-4400.

26. Gaussian 03, Revision A.1: Frisch, M. J.; Trucks, G. W.; Schlegel, H. B.; Scuseria, G. E.; Robb, M. A.; Cheeseman, J. R.; Montgomery, J. A., Jr.; Vreven, T.; Kudin, K. N.; Burant, J. C.; Millam, J. M.; Iyengar, S. S.; Tomasi, J.; Barone, V.; Mennucci, B.; Cossi, M.; Scalmani, G.; Rega, N.; Petersson, G. A.; Nakatsuji, H.; Hada, M.; Ehara, M.; Toyota, K.; Fukuda, R.; Hasegawa, J.; Ishida, M.; Nakajima, T.; Honda, Y.; Kitao, O.; Nakai, H.; Klene, M.; Li, X.; Knox, J. E.; Hratchian, H. P.; Cross, J. B.; Adamo, C.; Jaramillo, J.; Gomperts, R.; Stratmann, R. E.; Yazyev, O.; Austin, A. J.; Cammi, R.; Pomelli, C.; Ochterski, J. W.; Ayala, P. Y.; Morokuma, K.; Voth, G. A.; Salvador, P.; Dannenberg, J. J.; Zakrzewski, V. G.; Dapprich, S.; Daniels, A. D.; Strain, M. C.; Farkas, O.; Malick, D. K.; Rabuck, A. D.; Raghavachari, K.; Foresman, J. B.; Ortiz, J. V.; Cui, Q.; Baboul, A. G.; Clifford, S.; Cioslowski, J.; Stefanov, B. B.; Liu, G.; Liashenko, A.; Piskorz, P.; Komaromi, I.; Martin, R. L.; Fox, D. J.; Keith, T.; Al-Laham, M. A.; Peng, C. Y.; Nanayakkara, A.; Challacombe, M.; Gill, P. M. W.; Johnson, B.; Chen, W.; Wong, M. W.; Gonzalez, C.; Pople, J. A.; Gaussian, Inc., Pittsburgh PA, 2003.

(C) 2006 by MDPI (http://www.mdpi.org). Reproduction is permitted for noncommercial purposes. 